\title{
Laser-induced Crystalline-phase Transformation for Hematite Nanorod Photoelectrochemical Cells
}

\author{
Heejung Kong, ${ }^{\Delta, \dagger}$ Jinhyeong Kwon, ${ }^{\S, \dagger}$ Dongwoo Paeng, ${ }^{\perp}$ Won Jun Jung, $\mathbf{\nabla}$ Santosh Ghimire, ${ }^{\mathbf{\nabla}}$ \\ Joonghoe Dho, ${ }^{\nabla}$ Jae-Hyuck Yoo, " Sukjoon Hong, "Jinwook Jung, ${ }^{\ddagger}$ Jaeho Shin, ${ }^{\ddagger}$ Costas, P. \\ Grigoropoulos, ${ }^{\perp, *}$ Seung Hwan Ko, ${ }^{\star *}$ and Junyeob Yeo ${ }^{\Delta, \#, *}$
}

$\triangle$ Novel Applied Nano Optics lab, Department of Physics, Kyungpook National University, 80 Daehak-ro, Bukgu, Daegu 41566, Korea

\$Manufacturing System R\&BD Group, Korea Institute of Industrial Technology, 89

Yangdaegiro-gil, Ipjang-myon, Seobuk-gu, Cheonan, Chungcheongnam-do 31056, Korea

${ }^{\perp}$ Laser Thermal Lab, Department of Mechanical Engineering, University of California, Berkeley, CA 94720, USA

\ Department of Physics, Kyungpook National University, 80 Daehak-ro, Bukgu, Daegu 41566, Korea

" Physical and Life Sciences and NIF and Photon Sciences, Lawrence Livermore National Laboratory, Livermore, CA 94550, USA

'Department of Mechanical Engineering, Hanyang University, 55 Hanyangdaehak-ro, Sangnok-gu, Ansan, Gyeonggi-do 15588, Korea

¥Applied Nano and Thermal Science Lab, Department of Mechanical Engineering, Seoul National University, 1 Gwanak-ro, Gwanak-gu, Seoul 151-742, Korea

\#Department of Hydrogen and Renewable Energy, Kyungpook National University, 80 Daehak-ro, Bukgu, Daegu 41566, Korea

[*] To whom correspondence should be addressed.

E-mail: cgrigoro@berkeley.edu, maxko@snu.ac.kr, junyeob@knu.ac.kr

$[\dagger]$ Heejung Kong and Jinhyeong Kwon contributed equally to this work. 


\section{Measurement of charge separation and injection yields}

Charge separation yield $\left(P_{\text {sep }}\right)$ and charge injection yield $\left(P_{i n j}\right)$ were measured through the method reported by Dotan et al. that employs an electrolyte containing $\mathrm{H}_{2} \mathrm{O}_{2}$ as a hole scavenger. ${ }^{6}$ Theoretical maximum photocurrent $\left(J_{a b s}\right)$ of a photoelectrode is related with $P_{s e p}$ and $P_{i n j}$ by the following equations:

$$
\begin{aligned}
& J_{H 2 O}=J_{a b s} \times P_{\text {sep }} \times P_{i n j} \\
& J_{H 2 O 2}=J_{a b s} \times P_{s e p}
\end{aligned}
$$

, where $\mathrm{J}_{\mathrm{H} 2 \mathrm{O}}$ is net photocurrent measured in a $1 \mathrm{M} \mathrm{NaOH}$ aqueous solution and $\mathrm{J}_{\mathrm{H} 2 \mathrm{O} 2}$ is net photocurrent measured in a $1 \mathrm{M} \mathrm{NaOH}$ aqueous solution containing $0.5 \mathrm{M} \mathrm{H}_{2} \mathrm{O}_{2}$.

$J_{a b s}$ was calculated by the following integration:

$$
\int_{350}^{600 \lambda \cdot S(\lambda) \cdot \eta_{L H}(\lambda)} d \lambda
$$

, where $\lambda$ is wavelength, $S(\lambda)$ is solar irradiance (ASTM G-173-3), and $\eta_{L H}$ is light-harvesting efficiency depending on wavelength, which can be calculated from the following equation:

$$
\eta_{L H}(\lambda)=1-10^{-A(\lambda)}
$$

, where $A(\lambda)$ is absorbance depending on wavelength. ${ }^{7} \eta_{L H}$ of TAHN-550, TAHN-800, and LAHN-65 is shown in Figure S8 (c). The $P_{s e p} / P_{i n j}$-potential graphs of TAHN-550, TAHN800, and LAHN-65 are shown in Figure S10 (b)-(d), and the net photocurrent-potential graphs are shown in Figure S10 (e)-(g). 


\section{Figures}

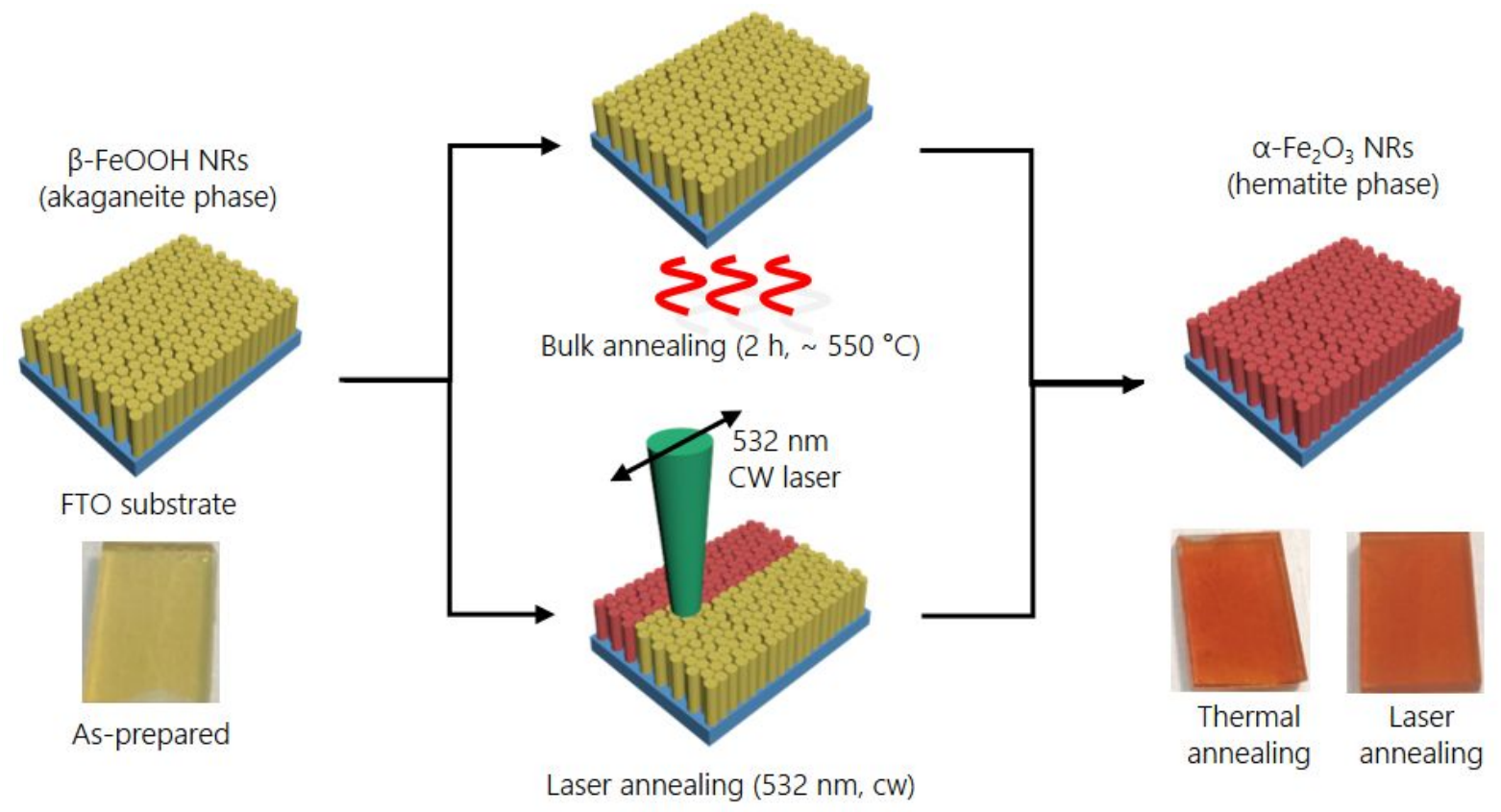

Figure S1. Schematic diagram. In the case of the conventional annealing process, the thermal annealing process is usually applied under $550{ }^{\circ} \mathrm{C}$ or $800{ }^{\circ} \mathrm{C}$ through drying oven after akaganeite NRs are grown on FTO substrate by hydrothermal growth. While, in the case of the laser annealing process (Laser-induced crystalline phase transformation, LIPT process), the continuous wave laser is applied as a local heat source and induce phase transformation to hematite. 


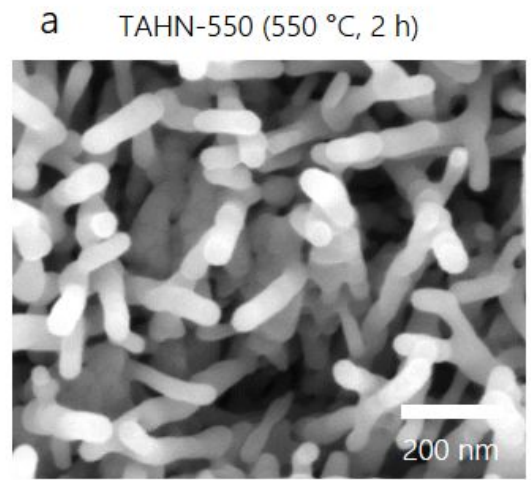

b $\operatorname{LAHN}\left(10 \mathrm{~mm} \mathrm{~s}^{-1}, 60 \mathrm{~mW}\right)$
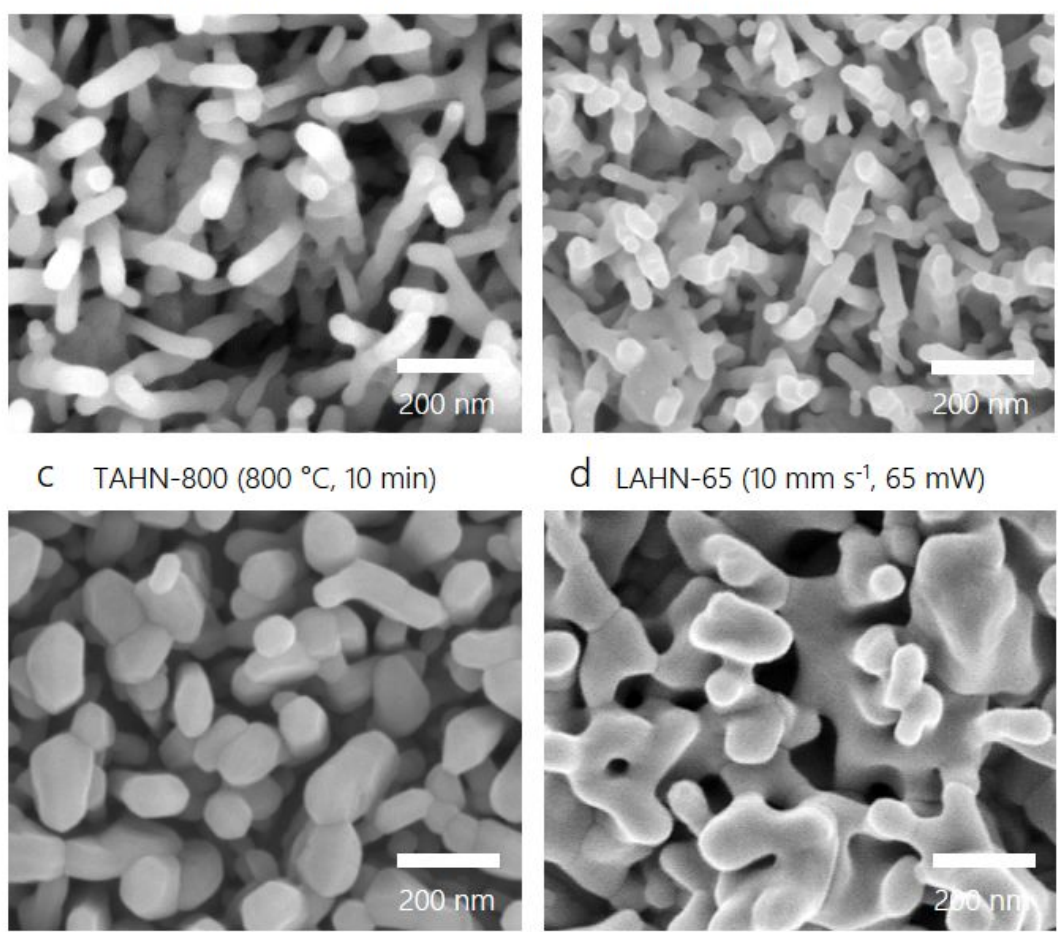

d LAHN-65 (10 $\left.\mathrm{mm} \mathrm{s}^{-1}, 65 \mathrm{~mW}\right)$

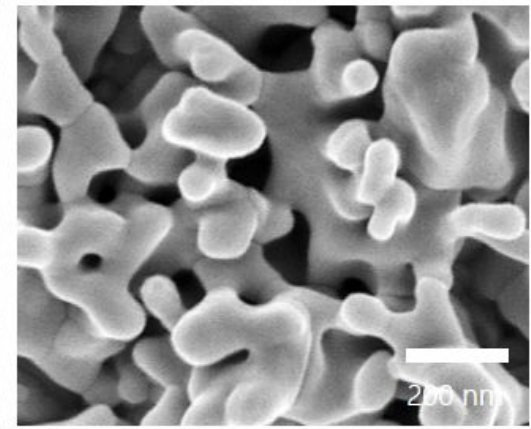

e
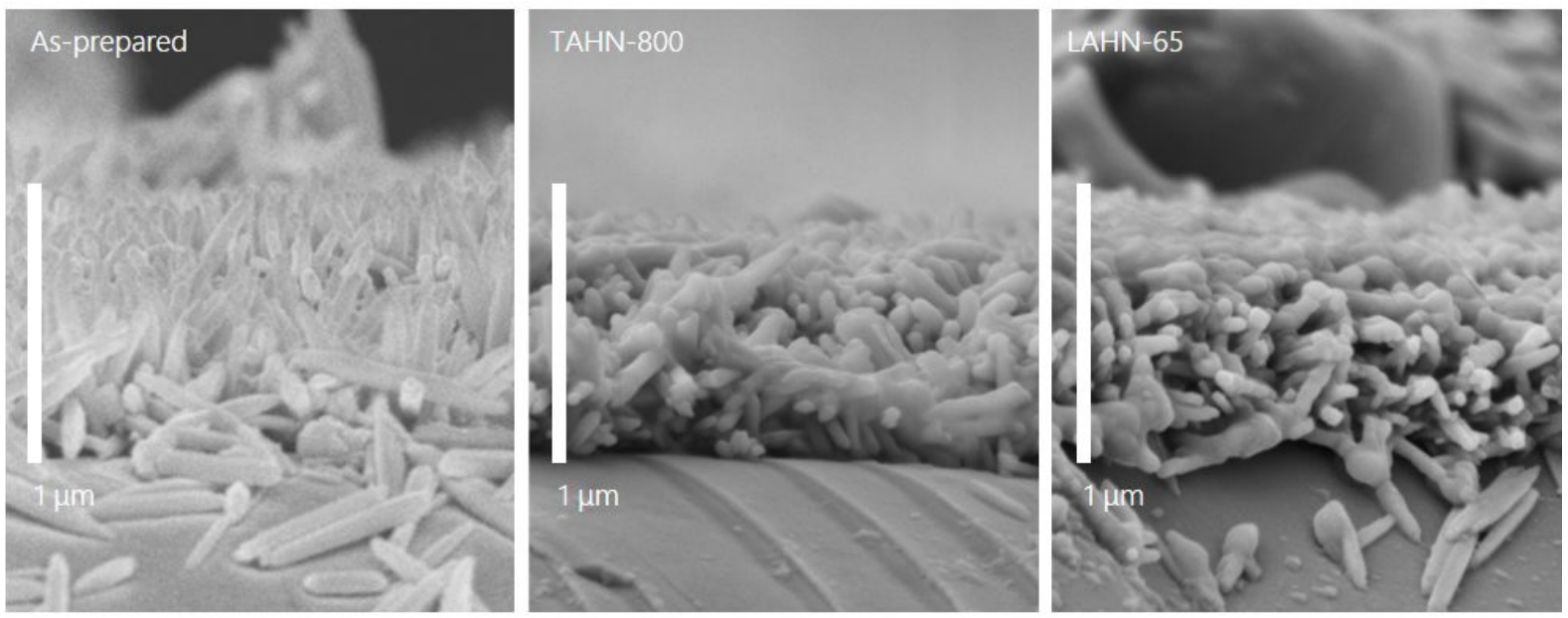

Figure S2. The magnified SEM images of (a) TAHN-550, (b) LAHN-60, (c) TAHN-800, (d) LAHN-65. The samples of (a) and (b) show a similar morphology. Likewise, the samples of (c) and (d) show a similar morphology. (e) The cross-section SEM images of as-prepared akaganeite NRs, TAHN-800, and LAHN-65. 
a

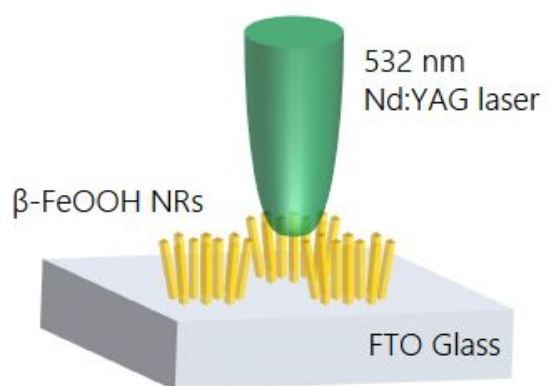

C Top focusing (10 $\mathrm{mm} \mathrm{s}^{-1}, 60 \mathrm{~mW}$ )

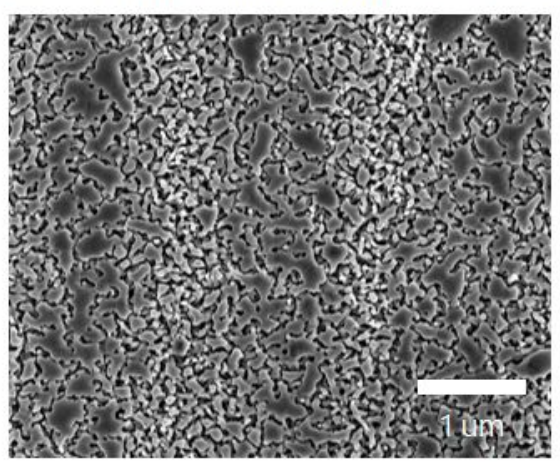

e

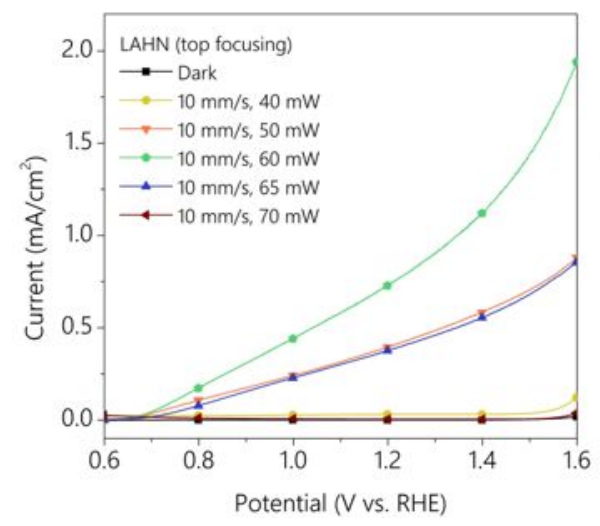

b Bottom focusing

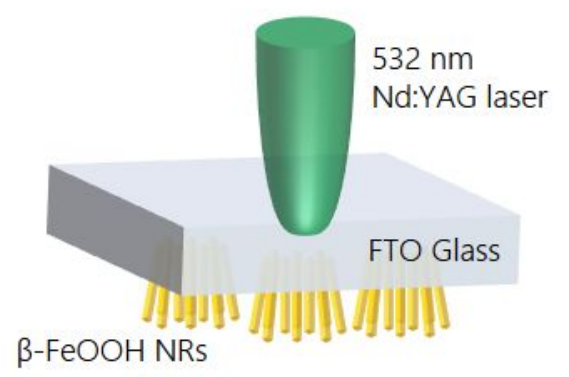

d Bottom focusing (10 $\mathrm{mm} \mathrm{s}^{-1}, 65 \mathrm{~mW}$ )

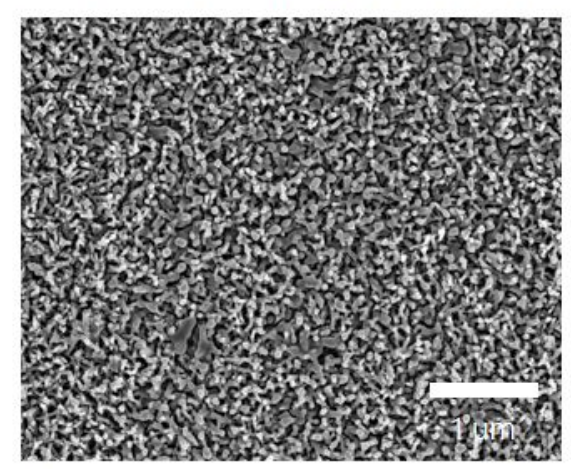

f

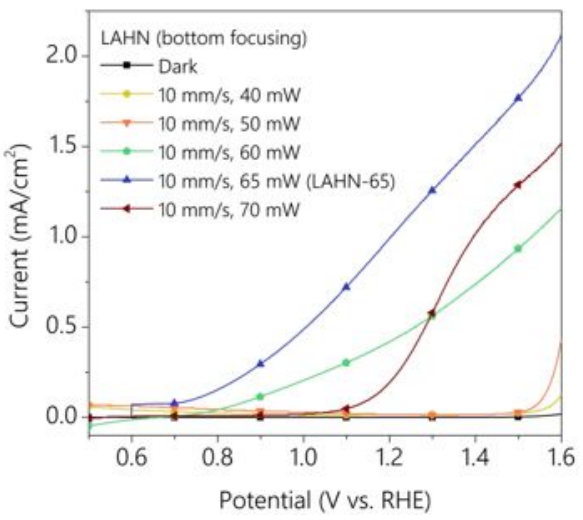

Figure S3. Top vs. bottom focusing techniques. (a)-(b) Schematic diagram of (a) top / (b) bottom focusing direction techniques. (c)-(d) The SEM images of the LAHN samples The LAHN samples employed top focusing (c) and bottom focusing (d) techniques at the same laser scan speed and power, respectively. (e)-(f) The PEC linear sweep curves of the LAHN samples with respect to the focusing techniques; (e) top and (f) bottom focusing techniques. The samples were fabricated by the LIPT process at various power $(40-70 \mathrm{~mW})$ at a fixed scanning speed of $10 \mathrm{~mm} \mathrm{~s}^{-1}$. 
a

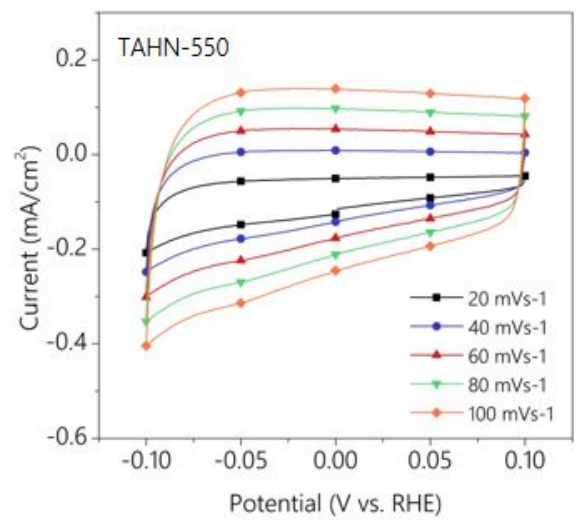

C

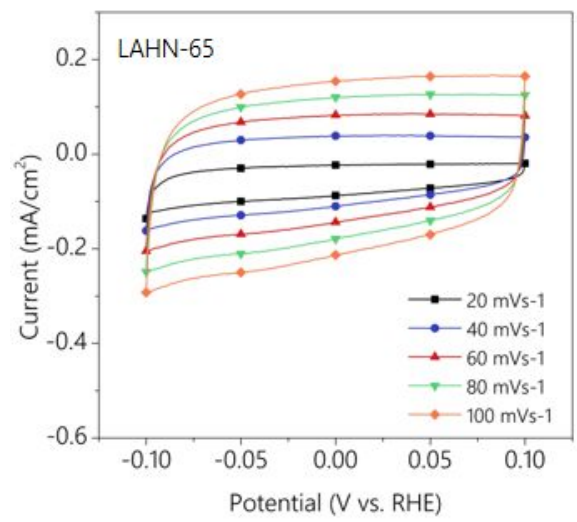

e

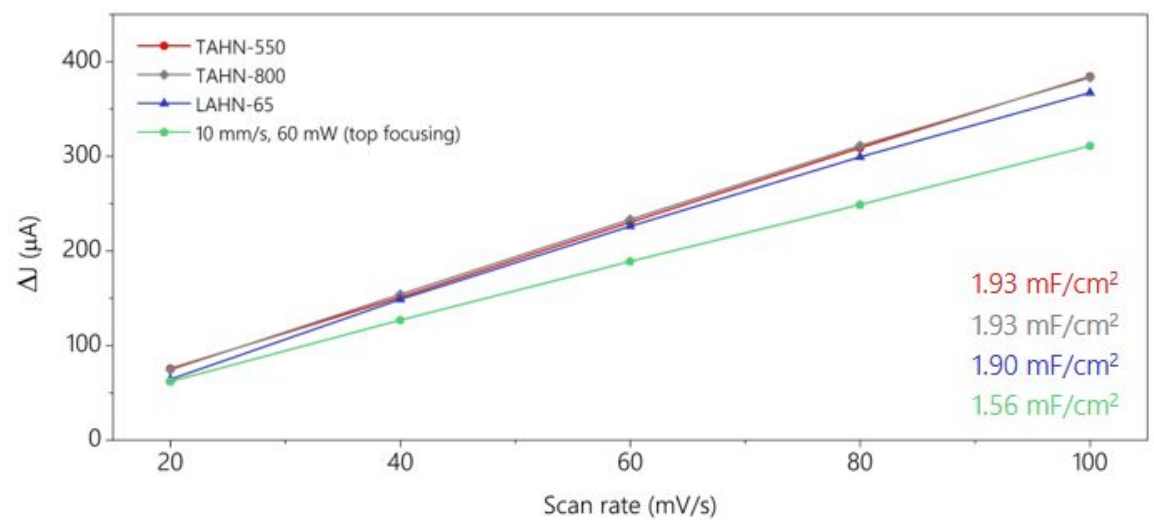

Figure S4. (a)-(d) The cyclic voltammetry (CV) curves of (a) TAHN-550, (b) TAHN-800, (c) LAHN-65, and (d) the top focusing LAHN sample $\left(10 \mathrm{~mm} \mathrm{~s}^{-1}, 60 \mathrm{~mW}\right)$, measured with respect to various scan rates $\left(20-100 \mathrm{mV} \mathrm{s}^{-1}\right)$. (e) Electrochemically active surface area (ECSA) of the samples. 

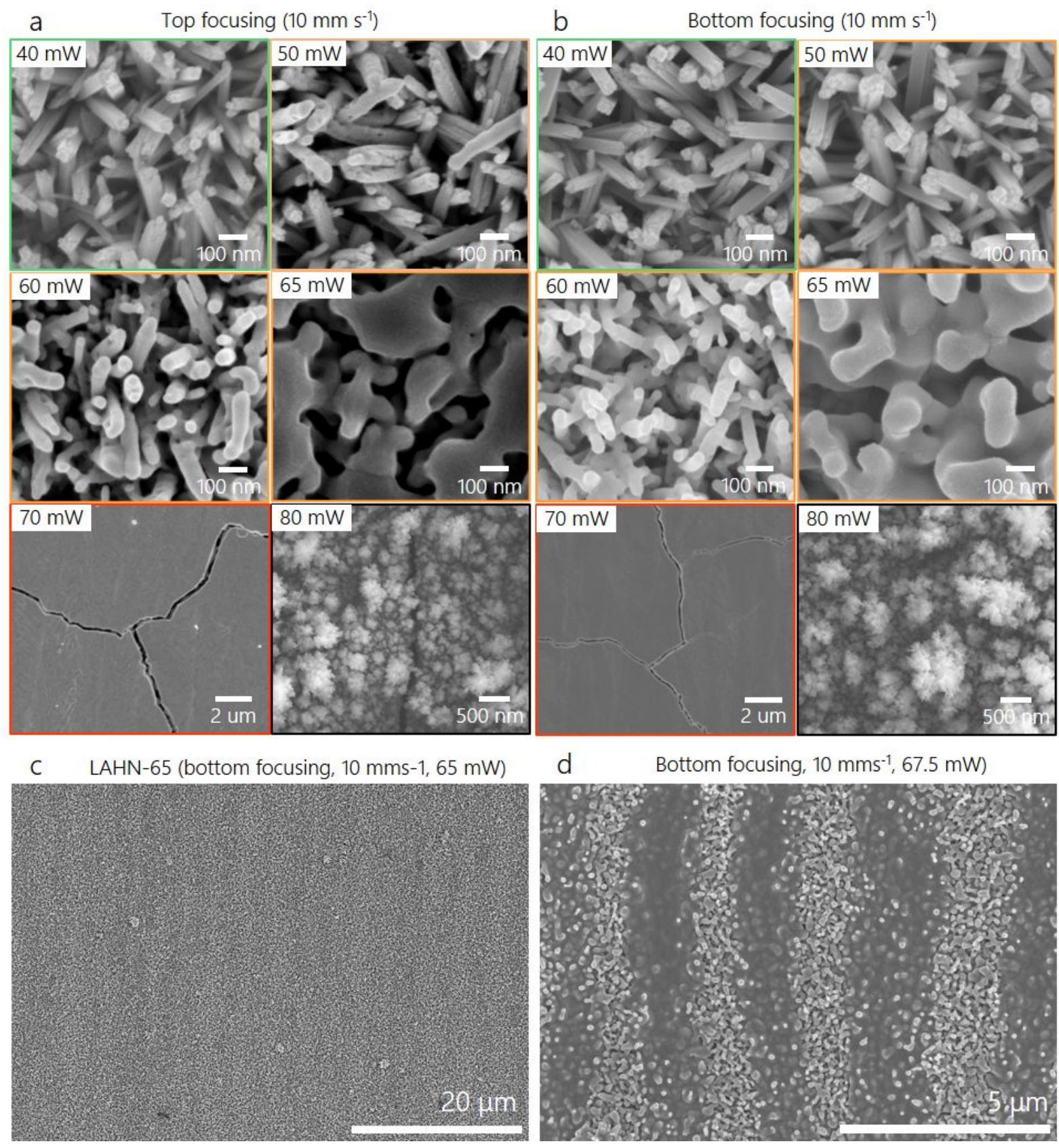

Figure S5. (a)-(b) The SEM images of combinatorial study for (a) top and (b) bottom focusing techniques at various laser power $(40-80 \mathrm{~mW})$. Green, orange, red and black boxes represent unchanged, slightly melted, excessively melted, and ablated hematite NRs, respectively. (c) The low magnification SEM image of LAHN-65. (d) The SEM image of the bottom focusing LAHN sample $\left(10 \mathrm{~mm} \mathrm{~s}^{-1}, 67.5 \mathrm{~mW}\right)$. 


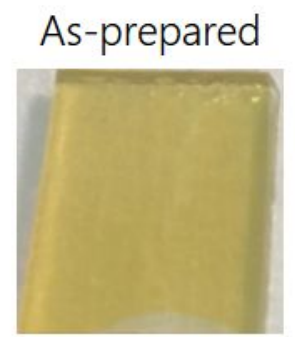

Laser
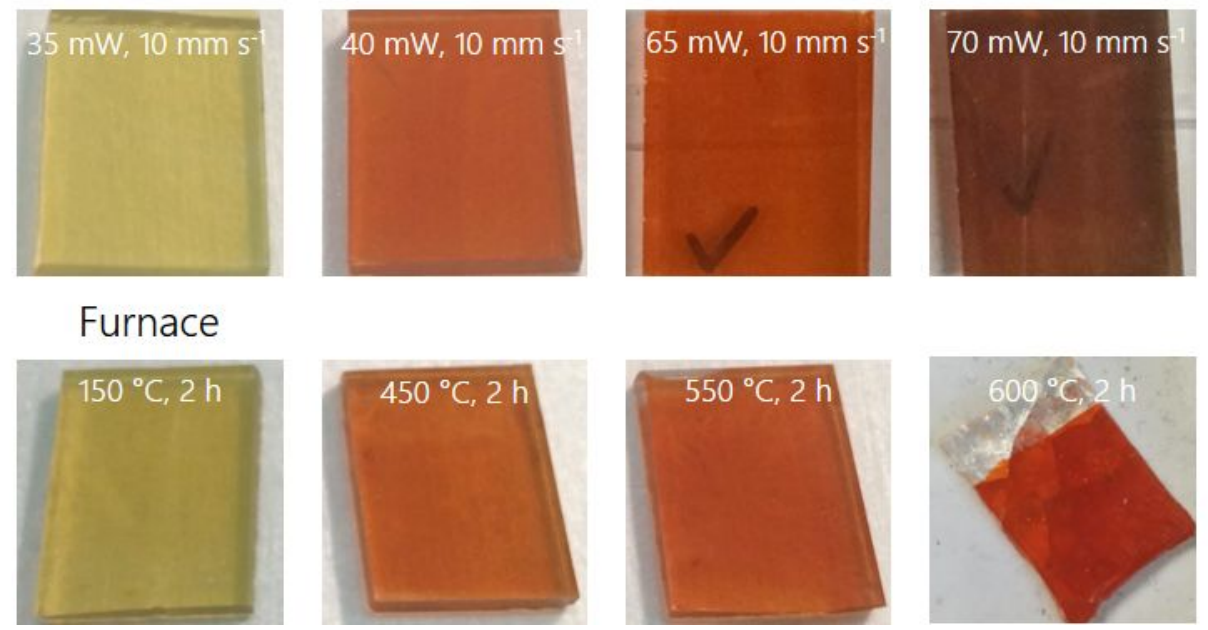

Figure S6. The digital images of as-prepared akaganeite NWs, the TAHN samples with different temperatures, and the LAHN samples of with different laser power. 
a

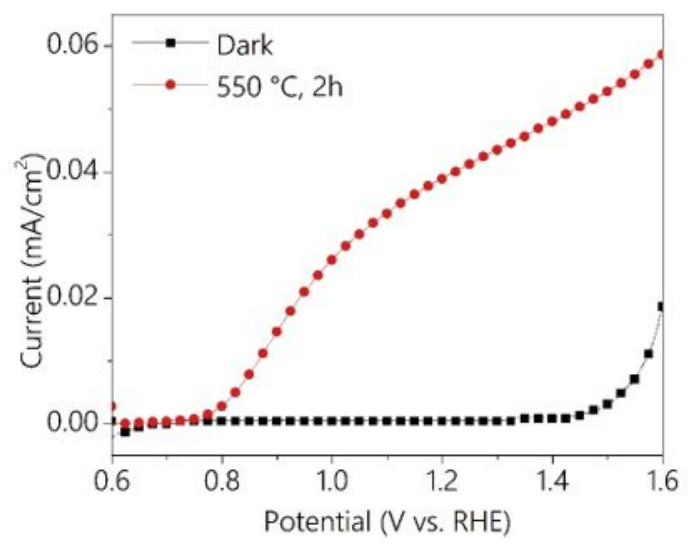

b

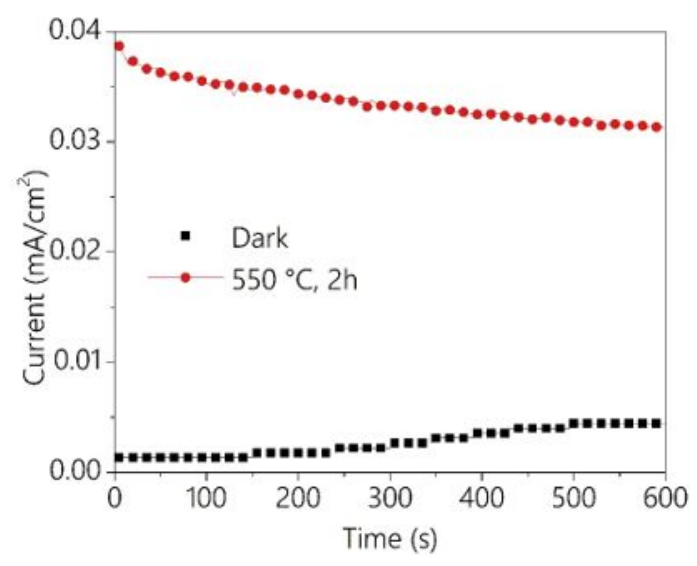

Figure S7. Photocurrent density as a function of (a) applied potential and (b) measurement time for the TAHN-550. 
a

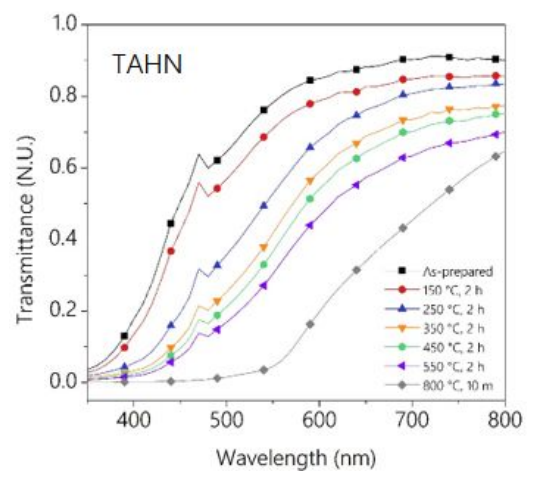

$\mathrm{b}$

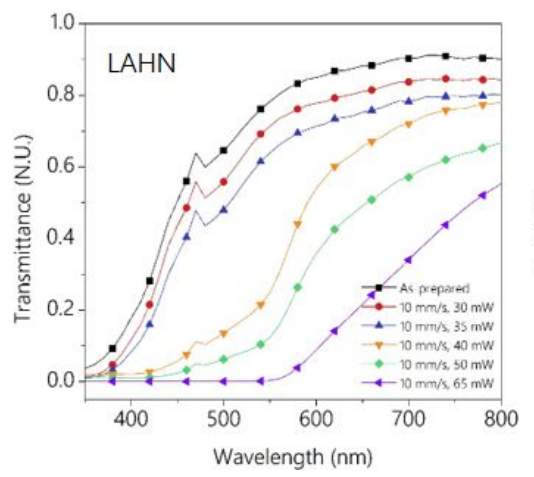

C

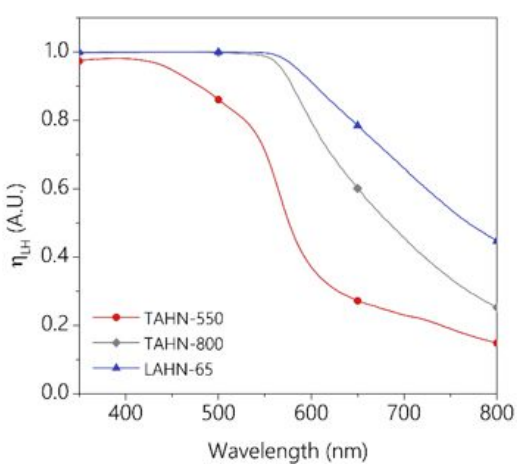

Figure S8. The optical properties of the hematite NRs. (a) The transmittance spectra of the TAHN samples annealed at various temperatures $\left(150-800{ }^{\circ} \mathrm{C}\right)$ and (b) the LAHN samples fabricated at various laser power $(30-65 \mathrm{~mW})$. (c) Light-harvesting efficiency $\left(\eta_{\mathrm{LH}}\right)$ spectra of TAHN-550, TAHN-800, and LAHN-65. 
a

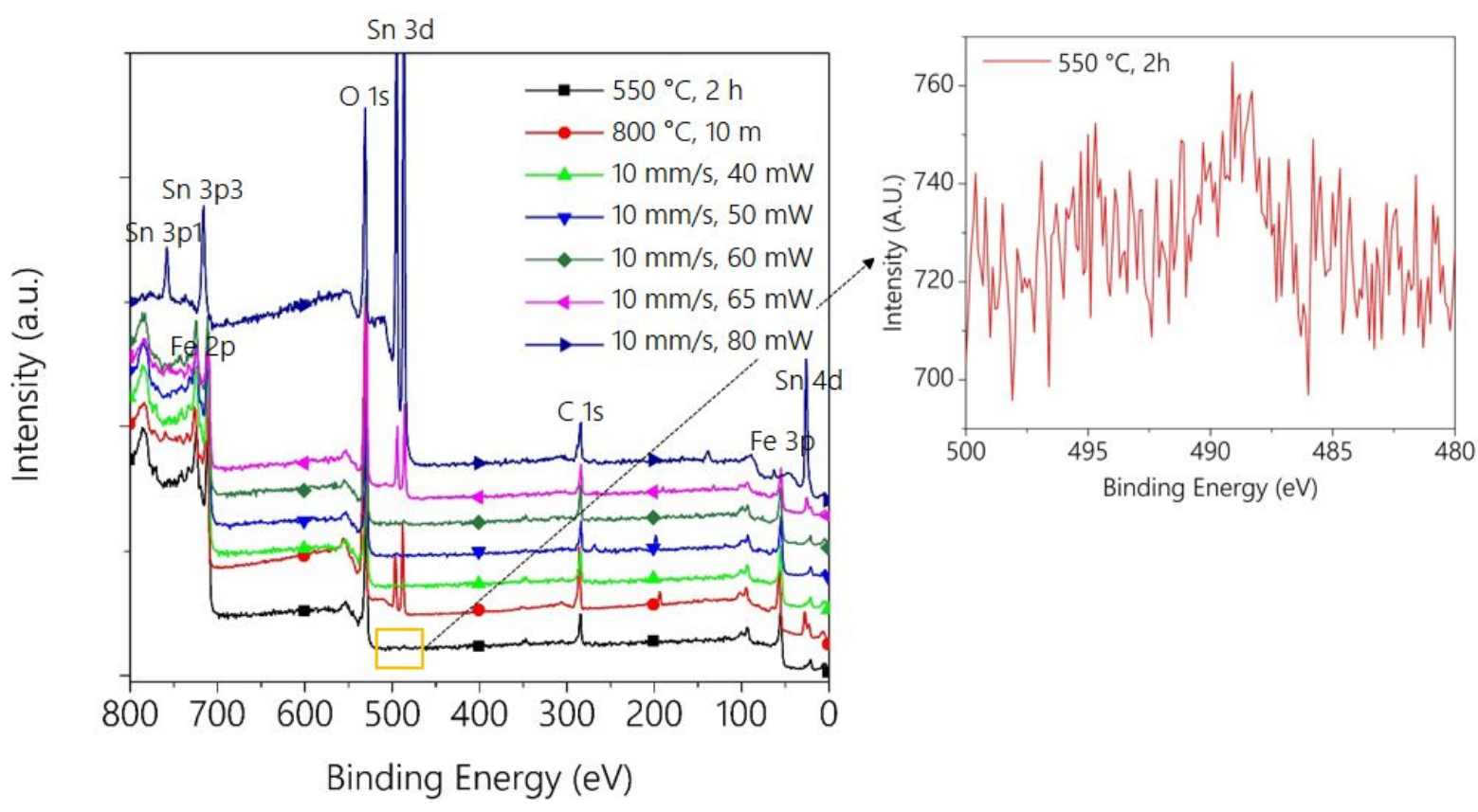

Figure S9. (a) The full XPS spectra for the TAHN-550, TAHN-800, and the LAHN samples fabricated with respect to various laser power at a fixed scan speed of $10 \mathrm{~mm} \mathrm{~s}^{-1}$. (b) The highresolution XPS spectrum in the Sn 3d region of the TAHN-550. 


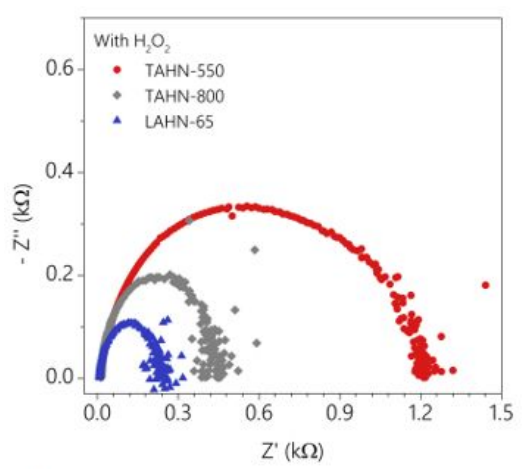

b

C

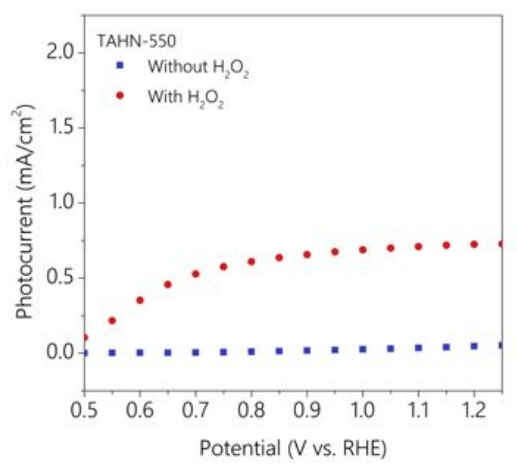

e

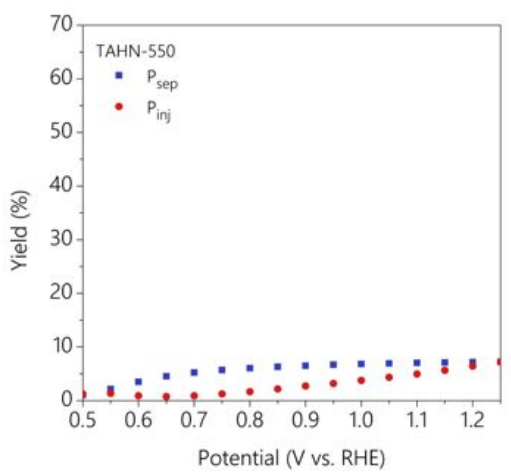

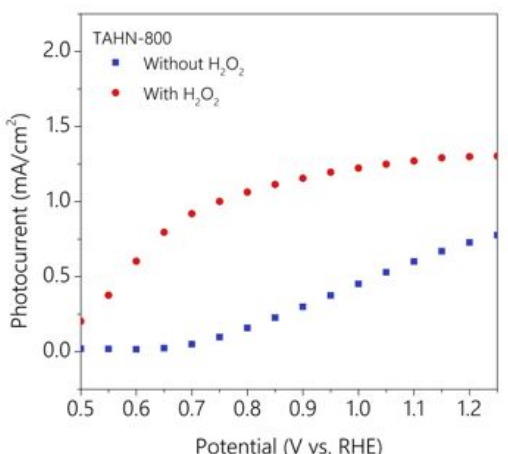

f

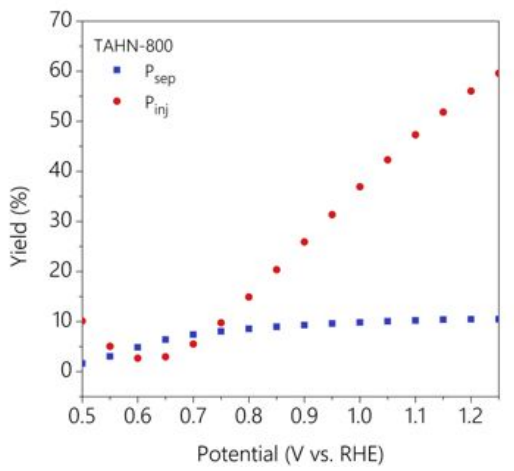

d

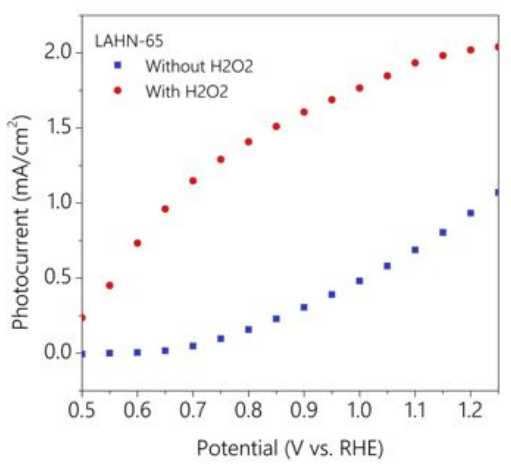

g

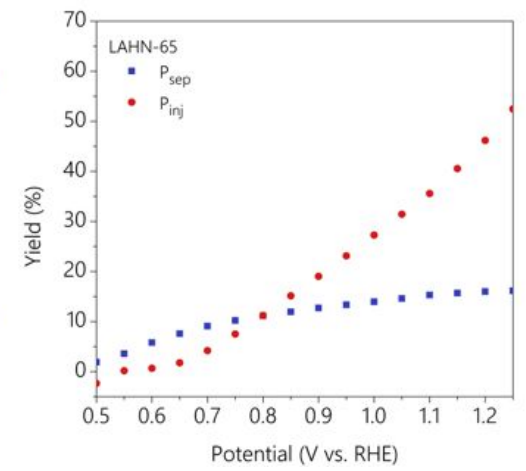

Figure S10. (a) Electrochemical impedance spectroscopy (EIS) spectra of TAHN-550, TAHN800 , and LAHN-65 in the $1 \mathrm{M} \mathrm{NaOH}$ aqueous solution containing $0.5 \mathrm{M} \mathrm{H}_{2} \mathrm{O}_{2}$ as a hole scavenger. (b)-(d) Net equilibrium photocurrent of (b) TAHN-550, (c) TAHN-800, and (d) LAHN-65, measured at various potentials in the $1 \mathrm{M} \mathrm{NaOH}$ electrolyte solution containing 0.5 $\mathrm{M}$ of $\mathrm{H}_{2} \mathrm{O}_{2}$. (e)-(g) Charge separation yield $\left(P_{\text {sep }}\right)$ and charge injection yield $\left(P_{i n j}\right)$ with respect to the applied potential (vs. RHE) of (e) TAHN-550, (f) TAHN-800, and (g) LAHN-65. 